\title{
Discussion on the Design Method of the Decorative Patterns of Yuan blue \& white
}

\author{
Lei Shi \\ Jingdezheng Ceramic Institute, Jiang Xi, Jingdezhen, 333001, China \\ Beifang University of Nationalities, Ningxia, Yinchuan, 750021, China
}

\begin{abstract}
Yuan blue \& white as the representative of Chinese porcelain, it is full of modeling design, dense decorative patterns, rigorous and orderly decoration layout, reflecting the perfect degree of Yuan porcelain craft from the production, decoration, painting to firing, won the favour of the world. Yuan blue \& white decorative patterns and rich and colourful decorative orderly layout, not only contains the profound Chinese nation cultural connotation, but also incorporates a number of Islamic decorative design rules. Yuan blue \& white decorative patterns and layout of the layout is carefully designed, in general, the reasonable arrangements pattern of the content and the overall control are dependent on the layout, if there is no reasonable layout of the global layout, it is impossible to form the Yuan blue \& white not chaotic, rigorous and orderly pattern decoration style, this special element blue \& white decoration layout is also a direct expression of a variety of cultural factors in the Yuan Dynasty, it is also the most intuitive. In this paper, taking the decorative pattern of the element blue \& white as the starting point to analyze the decorative patterns of the composition characteristics and design rules, through the in-depth study of this issue will help to understand the profound connotation of the cultural connotation.

Keywords: Yuan blue \& white, decoration, layout, design methods
\end{abstract}

\section{Introduction}

Mentioned Yuan blue \& white, people's minds appear is blue \& white, decorated with dense, full of Chinese porcelain shape. From the perspective of visual 
experience, the first impression of the person to remember is the most prominent feature of this thing, and Yuan blue \& white gives the impression that the shape of the outer wall is decorated with white blue flowers, although may not know the decorative What is the specific pattern, but still be able to feel its unique charm. Editor in the Chinese Association of Ceramics "History of Chinese ceramics" Yuan Qinghua decorative features summarized as: "Yuan blue \& white decorative features is that the level of multi-screen full, but because of proper handling, primary and secondary, seamless, does not give people a sense of trivial and piling." This article from the blue \& white modeling features, decorative techniques and spatial layout characteristics to analyze the Yuan blue \& white decoration layout design method.

\section{The spatial layout of Yuan blue \& white decorative design}

\subsection{The multi-layer layout}

Large blue \& white objects are generally decorated with a large number of objects arranged in a multi-level layout, whether it is bottles, cans of a class of Zhuo, or bowls, plates of a class of objects are based on modeling equipment changes, the use of Hyun lines for space separation.

Zuo generally from the mouth to the bottom foot table from top to bottom divided into multi-level decorative band, the theme of decoration generally arranged in the belly wide decorative tape, and mouth along the neck, shoulders, waist with a narrow, arranged auxiliary decoration. Round device, such as the general market from the bottom centre to the mouth along the disk from the inside out into multi-level decorative stripes, the theme of decoration generally arranged in a relatively flat disk heart, and mouth along the wall is arranged auxiliary decoration. There are individual decorative up to a dozen layers, can be described as airtight, and sometimes even some small or not easy to see the area is not miss, such as jade pot spring mouth along the mouth, the outer wall of the tape, covered animal ears Cans along the mouth part, etc., as well as covered with a cup of mug-shaped lid and covered with animal ear pots covered with a basinshaped lid is also divided into multi-level decoration.

In the multi-level layout design, if the different widths of the decorative bands with the classification analysis can be found in which the number of changes in the law: a combination of odd permutation; even permutations and combinations; have the use of the number of permutations arranged; Use of a ratio sequence. There is also one of the most special multi-level decorative pattern with decorative design methods.

\subsection{The full painting style layout}

Full-painted layout refers to the entire artifacts in addition to the mouth, foot and other parts of the painting is not painted or only painted with auxiliary decoration, while the vast majority of other instruments to complete a continuous panoramic 
picture painting. Full-painted layout of the decorative design methods are generally used for Zhuoqi. Round pieces of large objects are generally multi-level layout decoration, but there are some levels of separation is not particularly obvious, the same can be seen as full of decorative layout of the layout, there are some small pieces of objects such as disc, light class, Painting layout decoration. Although the full-painted layout decorative level is not much, but the ornaments occupy the objects of all effective visual decoration area, the overall effect is still dense.

\subsection{The scattered-style layout}

Scatter-point layout refers to the overall arrangement of objects in the decorative pattern, the pattern of the main part or the focus of the pattern to scattered scattered objects arranged in the whole body or a distribution in the overall arrangement of some of the more important patterns. Such as the British Museum of the staggered chrysanthemum pattern jade pot spring bottle, this jade pot spring bottle full of painting the whole table wrapped in chrysanthemum pattern, but if further observation, we found chrysanthemum chrysanthemum pattern is particularly prominent, , So that the form in the form of single-chrysanthemumbased scatter-point distribution, chrysanthemum arrangement free and easy, there is no specific law to follow. There are a number of bottom plates painted lotus pond mandarin duck pattern, banana leaf bamboo stone fruit map, but also the use of scattered-point layout of the decoration method for pattern arrangement.

\subsection{The suitable layout}

Suitable layout Decorative method mainly refers to the blue pattern of painting, according to the shape characteristics of the objects will be deformed to deal with changes in shape, so that the decoration and shape of unity. There are two forms of fit layout: one form is the theme decoration suitable for a specific geometric shape, known as the appropriate pattern, it is in a certain shape within the scope of the corresponding and independent of the complete decorative patterns, patterns of organization It is necessary to adapt to be decorative occasions or parts of the shape, with obvious characteristics of the fit. The other is the theme of decoration (such as cloud dragon or double phoenix pattern) suitable for decorative band or auxiliary decoration (such as the volume grass pattern, back to the end of the pattern, Pattern, sea wave pattern, etc.) to a continuous way for the two decorative band, the two sides are continuous patterns are strip, it is also known as the "striped pattern."

\subsection{The open-type layout}

Yuan blue \& white large objects, especially plum and gourd bottles in the multilevel layout decoration, but also popular in the main visual display area with flower-shaped opening to open up independent decorative space, usually four groups of flower-shaped opening, respectively, Theme of the decoration, to enhance the visual impact and composition of the rich. The general shape of the 
basic shape of the same shape to diamond, diamond-based, but because of the shape of objects and the shape of the space reserved for the different sizes, resulting in flower-shaped open flat and vertical long-shaped difference. There are individual markets also use this opening form, but the flower-shaped bottle, the tank variety, generally four-shaped. "According to research, the origin of the light is from Central Asia, West Asia Persian silverware and mosques architectural decorative elements of the impact.

For the decorative layout of the open-type layout, I believe that, in addition to the traditional sense of the flower-shaped opening, there is a similar to the "emission" -shaped form of composition. In short, is to look at the point of view from the broader market, or some cover with a lid canopy, because the petalshaped, wishful shoulder stripes or variant Lotus petal, in this particular angle, will form a new emission-like Flower-shaped, so this layout will be classified as open-type decorative approach.

This open layout of the decorative methods, mainly found in the broader market, according to the different decoration can be roughly divided into six kinds: the first petal-shaped to form a light-enclosed layout; the second to form a wishful shape to form a light-type layout; The third variant of the lotus petal to form a light-type layout, such a broader market in the heart of the plate, the use of six variants Lotus petal pattern combination of the formation of open layout, as is drawn from the disk centre, so variant Lotus The lower end of the grain is deformed into a sharp angle, which is different from the common form of the Lotus petal; the fourth one is the petal-shaped and the variant petal sharing, which forms the open-type layout. Lotus-shaped petals surrounded by the opening of the light, there are six variants of the outer periphery of the synthesis of an open flower petals, lotus petals for the leaf-shaped border decoration, very unique; the fifth petal-shaped and wishful sharing, The formation of the opentype layout of the enclosure, the plate with the heart of the petal-shaped combination of the formation of open-light design; the second layer to form a wishful shape to form a light; the third layer of the same shape to form a wishful shape enclosure light; Body lotus petals and wishful-shaped shared, surrounded by the formation of open-type layout, such a large plate set at the end of the heart painted six groups of white variants Lotus petal pattern and the centre of the white hyacinth combination like wheels, the outer ring to six groups of blue Ruyi cloud Shoulder composition, the opening layout of the use of the "white and blue $\&$ white" and "Green White" two decorative effects of painting. One of the most complex is undoubtedly the Iran National Museum of blue \& white flowers open Peony blueprint for the Phoenix Peony, the plate from the centre of the end of the large and small opening from the light of a total of six layers, this plate open light decorative pattern design Ruyi cloudy shoulder pattern, Variant Lotus petal pattern all use, open layout of the complex form, it is rare.

\subsection{The integrated layout}

Yuan blue \& white comprehensive layout, refers to the decoration layout design not only use a layout design method, but a variety of methods used in mixed, this 
is Yuan blue \& white decoration layout diversification, difficult to grasp the key. If there are multi-level and full-painted with the combination of the multi-level and the combination of open light; there are full-painted with the scatter-style combination; also full of painting with the appropriate combination, and even some multi-level layout, Open-type layout, suitable for layout and other layout decoration method. For the blue \& white decorative layout design method, according to a comprehensive analysis of various factors, such as a layout cannot be decorated with the main method to determine the layout of its classification, should be classified as integrated layout decoration method.

Use a variety of layout decoration methods in the same element blue \& white objects are many examples. Such as the blue \& white market are generally multilevel layout based on the combination of fit and open-type layout design; Yuan blue \& white plum bottle decorated with multi-level layout of the main, but also full of painted-style layout, on this basis, there Auxiliary ornaments narrow decorative band, the general will use the two sides of the continuous fit for the layout; and blue \& white large tanks to multi-level layout and full of paintedbased layout decoration-oriented, but in the mouth, shoulder and shin assistant Decoration is the use of two consecutive fit layout decoration, careful analysis is also a variety of layout decorative design.

\section{The use of Hyun pattern in the decoration pattern}

People appreciate the Yuan blue \& white and the general will pay attention to the changes in the shape and theme of decorative patterns, and often overlooked as a decorative space separation and boundaries of the Hyun lines. Hyun lines with fine lines that are used in the artifacts painted on the week, end to end, usually in the beginning of decoration for the object space separation and decorative patterns and space restrictions. Because of the use of the turntable for the description of hyacinth, the artifacts are often very structured, mainly in the shape of the object changes in the turning point, or as the theme of decoration and auxiliary decoration boundaries. In addition to a number of flat objects (such as the four-line flat pot and flat tube pot), pear-shaped pot, small pots, incense burners do not use Hyun lines, the remaining all blue \& white objects are almost all use.

Bottles, cans and other pieces of objects from the mouth to the end of the foot, from the top to the vertical separation of the layout of space, more than a dozen lines used Hyun lines, at least use one or two, mostly double Hyun pattern in the form, Between the two hyacinth lines naturally formed a white gap, so that each layer is more clear separation of decorative tape. Bowl, disk class device is mainly from the bottom to the edge of the mouth, drawn from the inside out of the concentric ring-hyun pattern for space area separation. 


\section{Conclusion}

In this paper, the decorative pattern design method of element blue \& white is discussed in detail, from the different shape and pattern layout design, to the specific spatial layout of the decorative method of a detailed analysis, focusing on the Yuan blue \& white decorative pattern layout design diversity and modeling, the richness of ornamentation is closely linked.

Yuan blue \& white decoration layout design, whether it is first according to the size of objects, shape the layout of the space division; or to determine the theme of the first decorative content, according to the decorative content and then space division? The author believes that the two situations are possible, a situation is: the special device-based Yuan blue \& white objects according to modeling features, according to the shape of the decorative area of different decorative space, and then according to the theme of the layout of the different layout of the specific layout style, such as Four-line flat pot, eight prism bottles, cans and so on. Another situation is: the conventional shape of the device is based on the theme of different decorative patterns, the first to determine the spatial location of the theme decoration, and then the rest of the space arrangement of other ornamentation, re-layout of the separation. Such as the story of the story lines and fish algae patterns as the theme of the decorative pattern of bottles, cans objects, may be the theme of the first decorative area of the area prior to the maximum visual area of the object left to the performance of more complex theme decoration, Of the optimal visual area. But no matter what kind of situation, the Yuan blue \& white decorative patterns in the layout design are unique, with the imprint of the times, deeply attracted us to explore the mysteries of which continue.

\section{References}

[1] Chinese Silicate Society editor. "Chinese Ceramics History". Beijing: Cultural Relics Press, pp.68-70, 1982

[2] Shanghai Museum, "Blue and white century: yuan blue and white and Yuan Dynasty history, art, archeology". Beijing: Peking University Press, pp.87- 91, 2013

[3] "The history of arts and crafts". Shenyang: Liaoning Education Press pp. 1820, 1999

[4] Cao Hui. "Aesthetic Study of Visual Form". Beijing: People's Publishing House, pp.58- 61, 2009 